\title{
NUMERICAL STUDY FOR CENTRIFUGAL MODEL TESTS OF A SINGLE PILE FOUNDATION INSTALLED IN SANDY DEPOSITS
}

\author{
C. W. Lu* \\ Department of Construction Engineering \\ National Kaohsiung First University of Science and Technology \\ Kaohsiung, Taiwan 91164, R.O.C.
}

\begin{abstract}
It is believed that a dynamic analysis is urgently required to provide a more reliable numerical method for seismic evaluation of a full system, which includes foundation, super structure, and ground in earthquake zones such as Taiwan and Japan. A centrifugal model test of pile foundation is simulated numerically using a three-dimensional finite-element model (3D-FEM) code in this study. In the numerical simulation, parameters of the sandy soils in tij model that are derived from accumulated experiences in static tests are first calibrated by centrifugal vibration tests of sandy ground. Model tests of a single pile foundation installed in grounds of same unit weight of soil as in the static tests are then simulated using the calibrated parameters. The numerical simulation resulted in a good agreement with the corresponding physical model tests. By comparing the computed and the observed results, one can find and confirm that it is necessary to employ an appropriate soil model to reproduce dynamic soil behavior due to major vibration. Representation of pile by beam element in the numerical analysis is applicable when attention is paid on the response acceleration of top of pile foundation, on soils at some distances to the pile foundation, and on bending moment of the pile in a stiffer ground. Equal-displacement boundary condition for two-side boundaries is proven to be efficient. To reduce the computation time, the assumption of a constant damping of viscous matrix is acceptable.
\end{abstract}

Keywords : Dynamic analysis, Pile foundation, 3D-FEM, Centrifugal model test.

\section{INTRODUCTION}

Seismically loaded piles are designed to withstand not only the inertial forces generated from the oscillation of the super structure but also the deformations of a ground caused by the passage of seismic waves through the surrounding soil. It was noted that structural researchers often tend to use a too simplified model dealing with ground and that geotechnical researchers tend to use a too simplified model dealing with super structures. It is believed, however, that a dynamic analysis is urgently required to provide more reliable simulations for the evaluation of a full system, which includes foundation, super structure, and ground, especially in the earthquake zones such as Japan and Taiwan. Unfortunately, dealing with the full system with a dynamic analysis was usually thought to be difficult when the nonlinearity of both soil and structure must be taken into account. Few studies have been done in this field through either experiments or numerical analyses.

Some methods for direct seismic analysis of pile foundations were based on linear elastic behaviors, such as EI-Marsafawi et al. [1] who conducted a complete elastic analysis for pile foundation using 3D boundary element formulations, in which nonlinear behavior of soil and pile are not taken into account. Anandarajah et al. [2] conducted a 2D finite element analysis to simulate a pile-supported two-story structure under earthquake shaking by using the isotropic bounding surface model to describe the stress-strain relation of the soils. Fukutake et al. [3] considered 3D finite element analysis of the soil behavior described by the RambergOsgood model with effective stress conditions. Finn and $\mathrm{Wu}$ [4] developed a quasi 3D method to reproduce the dynamic nonlinear behavior of pile foundations. Due to the limitations of the constitutive models adopted for soils, additional assumptions were made by Finn and $\mathrm{Wu}$ to compensate for the insufficiency of the models, e.g., tension cutoff and shearing cutoff were applied in order to describe the gapping between the soil and the piles. Wakai et al. [5] conducted a seismic analysis of a bridge-ground system using a 3D nonlinear dynamic analysis based on a total stress with a simplified soil model. Immura et al. [6] conducted a series of centrifuge model tests for understanding the seismic behaviors of pile groups. Lin et al. [7] evaluated lateral performance via inclinometer data and comparing his proposed methodology with other methods in a static condition. Tokimatsu et al. [8] studied effects of inertial and kinematic interaction on seismic behavior of

* Assistant Professor 
pile foundation by using both p-y curve and experimental work involving a discrete spring, which, however, is conventionally thought to be insufficient to reproduce behavior of soils. Finn et al. [9] used a finite element model for soil-pile interaction to simulate experiment results of a shaking table test with restrictions on the motion in the vertical direction and in the direction to that of the pile motion. Kimura and Zhang [10] conducted a series of static and dynamic 3D elastoplastic finite element analyses on a simplified sway-rocking model (S-R model) and on a full system to investigate the dynamic behavior of group-pile foundation during earthquake. Zhang et al. [11] numerically simulated a field test of a real-scale 2-pile foundation subjected to lateral cyclic loading up to ultimate state with a 3D elastoplastic finite element analysis, taking into consideration the influence of different constitutive models adopted for soils. Suggestions for the effect of piled foundation seismic behaviors due to different soil models were also discussed by Ye et al. [10].

Aforementioned papers pointed out the importance of an advanced model in dynamic numerical analysis and the need of a reliable numerical tool. However, these papers were mostly of either pure numerical study or numerical simulation on one or two case histories. To show applicability of a numerical model on simulating dynamic behavior of soil-pile system, a parametric study of the numerical model or a simulation on case histories by the numerical tool is thought to be insufficient, and a more rigorous process for numerical model calibration and verification is needed.

In this paper, a calibration and verification process that examines the performance of a numerical code was proposed. First of all, two types of physical model, one using grounds that consist of Toyoura sand and the other using a full system that consists of a mass loaded single pile in grounds, were introduced for the study. Next, a 3D-FEM code, which uses tij soil model for reproducing nonlinear soil behavior and beam element for representing pile behavior, was employed to simulate a series of centrifugal model tests. Finally, the accuracy of the soil parameters obtained from accumulated experiences on the Japanese standard sand and the consistency of the numerical code were first confirmed by simulating the centrifugal tests of grounds-only model, and then the numerical tool was verified by comparing the simulation results on single pile foundation in different grounds to the observed results from the physical model tests. This paper presented the effectiveness of numerical techniques and applicability of the soil model by comparing the computed and the observed model tests in a more rigorous procedure.

\section{SOIL MODEL}

Two types of physical models and two unit weights of soil are considered in this paper. One type is grounds that are made of Toyoura sand, and the other type is a full system that consists of a mass loaded sin- gle pile in grounds of the same sand. The two unit weights of soil are $14.3 \mathrm{kN} / \mathrm{m}^{3}$ (i.e. loose) and $15.8 \mathrm{kN} / \mathrm{m}^{3}$ (i.e. dense). The four simulation cases are illustrated in Fig. 1. For convenience sake, the two grounds-only models of unit weights $14.3 \mathrm{kN} / \mathrm{m}^{3}$ and $15.8 \mathrm{kN} / \mathrm{m}^{3}$ are referred to as Ground I and Ground II, respectively, and the two full-system models of unit weights $14.3 \mathrm{kN} / \mathrm{m}^{3}$ and $15.8 \mathrm{kN} / \mathrm{m}^{3}$ are referred to as Single pile I and Single pile II, respectively. All sands in the ground are dry in the physical model; therefore, a total stress analysis is used here.

Strength and dilatancy of soils are dominated by the stress and strain increment variables. The stress and strain relationship controls the performance of soils that includes passage of waves through this soil medium. Cam-clay like models that use extended Mises strength criterion is not able to account for the effect of intermediate principal stress properly, and unrealistic strength under generalized three-dimensional stresses is given except for triaxial compression condition. Nakai [13] proposed a modified stress tensor (tij) based on the Spatially Mobilized Plane (SMP) concept that uses Matsuoka-Nakai strength criterion to consider the influence of intermediate principal stress on the strength and the dilatancy of soils. Matsuoka-Nakai strength criterion is a convex surface on the $\pi$-plane in the ordinary stress space and satisfies Mohr-Coulomb criterion under axisymmetric conditions (triaxial compression and extension).

The soil model is characterized mainly by the introduction of a modified stress tensor tij and the formation of a plastic work-like quantity, which forces the flow rule to be given in the modified stress space. Invariants $\left(t_{N}, t_{S}\right)$ of the modified stress tensor lead to the failure criterion $\mathrm{I}_{1} \mathrm{I}_{2} / \mathrm{I}_{3}=$ constant, where $t_{N}$ represents the normal stress and $t_{S}$ the shear stress. This strength criterion satisfies Mohr-Coulomb criterion under axisymmetric conditions. To express stress path dependency of the plastic flow, plastic strain increment is decomposed into two components, of which one is governed by the flow rule and the other is isotropic and

\begin{tabular}{|c|c|c|}
\hline & Loose sand & Dense sand \\
\hline $\begin{array}{c}\text { Ground } \\
\text { only }\end{array}$ & 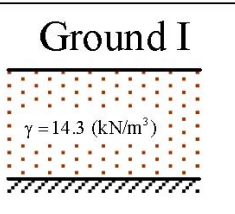 & 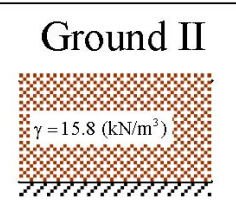 \\
\hline $\begin{array}{l}\text { Ground+ } \\
\text { pile+ } \\
\text { super } \\
\text { structure }\end{array}$ & \begin{tabular}{l} 
Single pile I \\
\hdashline \\
\hdashline \\
$\vdots$ \\
$\vdots$ \\
$\vdots$ \\
\hdashline
\end{tabular} & 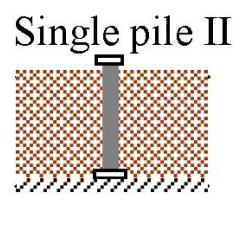 \\
\hline
\end{tabular}

Fig. 1 Four simulation cases 
produced only when the normal stress $t_{N}$ increases, and plastic volumetric strain is used as the hardening variable. For modeling sand, a fabric anisotropy tensor is incorporated to express inherent anisotropy, and plastic work is used as the hardening variable. This soil model is able to satisfactorily account for influence of intermediate principal stress on the deformation and strength of soils, dependency of plastic strain directions on strain path, and positive dilatancy during strain hardening.

The parameters in this soil model include $R_{f}$ (stress at failure), $\lambda$ (compression index), $k$ (swelling index), $D_{f}$ (gradient of strain ratio at critical state), $\alpha$ (stressdilatancy parameter), $m$ (ratio of volumetric strain to mean effective stress), $v$ (Poission's ratio), $e_{0}$ (Void ratio), and $\rho$ (soil density). $v, \lambda, k, R_{f}, m$ and $D_{f}$ can be obtained by using conventional triaxial compression tests, where the first four parameters are the same as those in Cam-clay model, and $\alpha$ is obtained by trial and error to get the best fit between the measured results of the stress-dilatancy curve for different stress paths. Detailed discussion on how to determine these parameters can be referred to corresponding references [13 15]. The values of the parameters, determined from the accumulated experiences on Toyoura sand [15,16], are provided in Table 1.

\section{PHYSICAL MODEL TESTS}

Geotechnical centrifuge tests have been observed to be very effective means for investigating problems in soil mechanics under the prototype stress conditions proposed by Schofield [17], Scott [18], Prevost and Scanlan [19], and others. Facility for the model tests in this paper is developed by Mitsubishi Heavy Industry in Japan. The arm radius of the centrifuge to the basket platform is $2.5 \mathrm{~m}$ for dynamic tests. An electromagnetic shaking table of $369 \mathrm{~mm}$ in height, $510 \mathrm{~mm}$ in length, and $330 \mathrm{~mm}$ in width is put on the centrifuge simulator. The laminar container consists of rectangular hollow frames made of square steel tubes. Linear bearings are installed to reduce friction during shaking.

For the two unit weights of $14.3 \mathrm{kN} / \mathrm{m}^{3}$ and $15.8 \mathrm{kN} / \mathrm{m}^{3}$, dry Toyoura sand is arranged to have relative densities of $30 \%$ and $73 \%$ and void ratios of 0.85 and 0.68 , respectively, in the container for grounds in all physical model tests. To present the mass on a superstructure for single pile foundation model, $45 \mathrm{~mm} \times$ $45 \mathrm{~mm} \times 20 \mathrm{~mm}$ SUS (Stainless Used Steel) block weighing $273 \mathrm{~g}$ is fixed at the top. The aluminum pile of $386 \mathrm{~mm}$ in length, $20 \mathrm{~mm}$ in diameter, and $1 \mathrm{~mm}$ in thickness is used in the physical model test for a single pile foundation. The bending stiffness of pile is $191.2 \mathrm{~N}-\mathrm{m}^{2}$. The pile is installed down at the bottom of the container, of which horizontal movements of the toe is fixed. Table 2 shows the dimensions of physical model and its corresponding prototype.
Table 1 Material parameters of ground

\begin{tabular}{|c|c|c|c|c|c|c|c|c|c|}
\hline Soil & Title & $\nu$ & $\mathrm{D}_{\mathrm{f}}$ & $\mathrm{m}$ & $\alpha$ & $\mathrm{e}_{0}$ & $\mathrm{R}_{\mathrm{f}}$ & $\lambda\left(\mathrm{C}_{\mathrm{t}}\right)$ & $\kappa\left(\mathrm{C}_{\mathrm{e}}\right)$ \\
\hline $\begin{array}{l}\mathrm{Dr}= \\
30 \%\end{array}$ & Ground I & 0.30 & -0.6 & 0.3 & 0.85 & 0.85 & 4.05 & 0.01045 & 0.004295 \\
\hline $\begin{array}{l}\mathrm{Dr}= \\
73 \%\end{array}$ & Ground II & 0.30 & -0.6 & 0.3 & 0.85 & 0.68 & 4.05 & 0.0095 & 0.0039 \\
\hline
\end{tabular}

Table 2 Parameters of single pile foundation

\begin{tabular}{|c|c|c|c|c|}
\hline \multicolumn{2}{|c|}{ Items } & Unit & Physical model & Prototype \\
\hline \multirow{4}{*}{ SUS block } & Dimension & & $45 \mathrm{~mm} \times 20 \mathrm{~mm}$ & $2.025 \mathrm{~m} \times 0.9 \mathrm{~m}$ \\
\cline { 2 - 5 } & Mass & $\mathrm{M}$ & $267 \mathrm{~g}$ & $24.3 \mathrm{ton}$ \\
\cline { 2 - 5 } & Elevated height & $\mathrm{L}_{1}$ & $120 \mathrm{~mm}$ & $5.4 \mathrm{~m}$ \\
\cline { 2 - 5 } & Material & - & SUS & - \\
\hline \multirow{4}{*}{ Pile } & Diameter & $\mathrm{D}$ & $20 \mathrm{~mm}$ & $0.9 \mathrm{~m}$ \\
\cline { 2 - 5 } & Thickness & $\mathrm{t}$ & $1 \mathrm{~mm}$ & $45 \mathrm{~mm}$ \\
\cline { 2 - 5 } & Embedded length & $\mathrm{L}_{2}$ & $369 \mathrm{~mm}$ & $16.6 \mathrm{~m}$ \\
\cline { 2 - 5 } & Stiffness & EI & $191.2 \mathrm{~N} \cdot \mathrm{m}^{2}$ & $7.842 \times$ \\
$10^{8} \mathrm{~N} \cdot \mathrm{m}^{2}$ \\
\cline { 2 - 5 } & Material & - & Aluminum & - \\
\hline
\end{tabular}

The same physical model tests conducted by Oogawa et al. [20], in which $45 \mathrm{~g}$ centrifugal acceleration was applied on the in-flight shaker with seismic input, are used in the study. Measurements consist of the response acceleration in Ground I and II and the response acceleration and the bending moment in Single pile I and II. The locations of the gages on piles and grounds are illustrated in Figs. 2 and 3. The response accelerations in Ground I and II are measured by ACC4, ACC 3 , and ACC2 located at $31 \mathrm{~mm}, 133.5 \mathrm{~mm}$, and $209 \mathrm{~mm}$ beneath the ground surface in the physical model, respectively, as shown in Fig. 2(a) and at the center of the plane view of ground as shown in Fig. 2(b). On the other hand, the response accelerations in Single pile I and II are measured by GA5, GA4, GA3, and GA2 located at $28 \mathrm{~mm}, 75 \mathrm{~mm}, 128 \mathrm{~mm}$, and $188 \mathrm{~mm}$ beneath the ground surface, respectively, and the strain gages BM4, BM3, BM2, and BM1 are installed in between those locations as shown in Fig. 3(a) to measure the bending moment. Figure 3(b) illustrates the dimensions of the SUS block installed at the top of single pile foundation.

The seismic input, recorded at Port-island of Kobe during the Hyogoken-Nambu earthquake in Japan, used on the shaker is shown in Fig. 4. This seismic wave is also employed in the numerical simulation introduced in the next section. The maximum acceleration of this seismic event is $687 \mathrm{gal}$ in a horizontal direction.

\section{NUMERICAL SIMULATION}

A series of dynamic analysis of the physical models using 3D numerical modeling is conducted to simulate the mechanical behavior in a seismic event. A direct integration method of Newmark- $\beta$ is adopted. The 


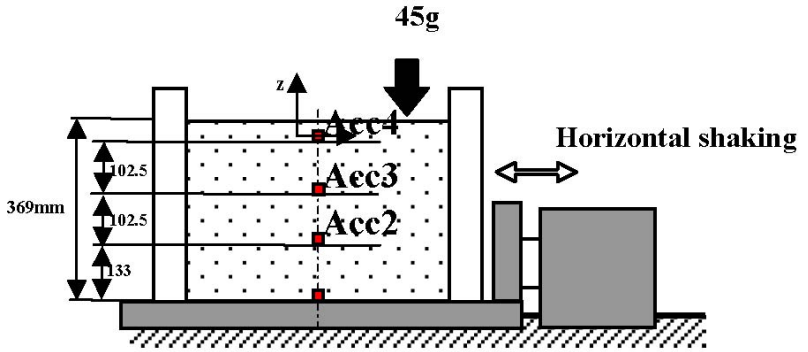

2(a) Layout of shear box in centrifuge test (Ground I and II)

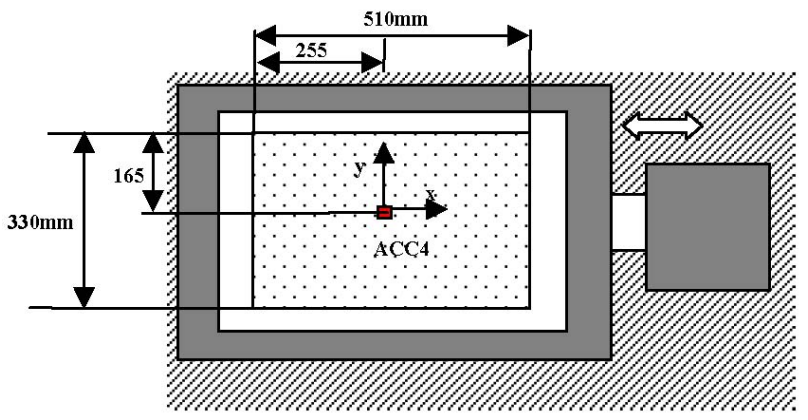

2(b) Plane view of shear box in centrifuge test (Ground I and II)

Fig. 2 Physical model of Ground I and II

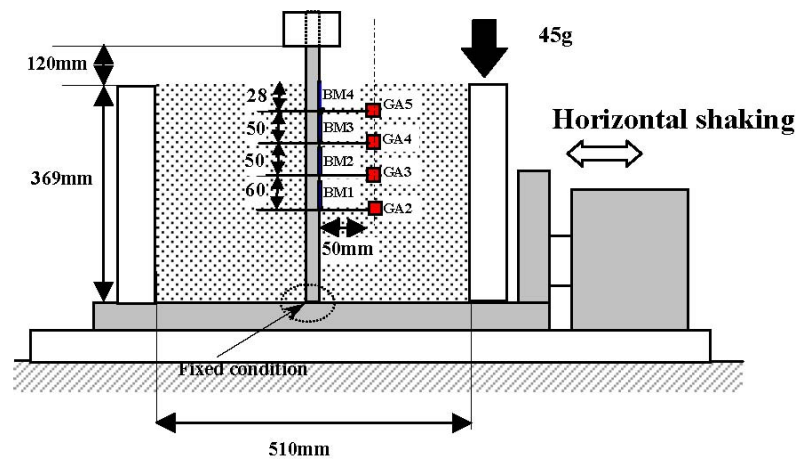

3(a) Layout of the shear box of Single pile I and II

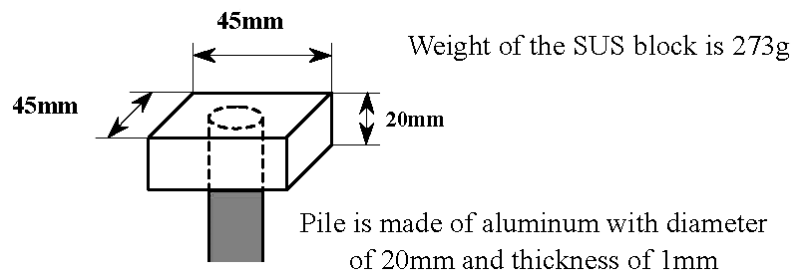

3(b) Layout of the pile top of Single pile I and II

Fig. 3 Physical model of Single pile I and II

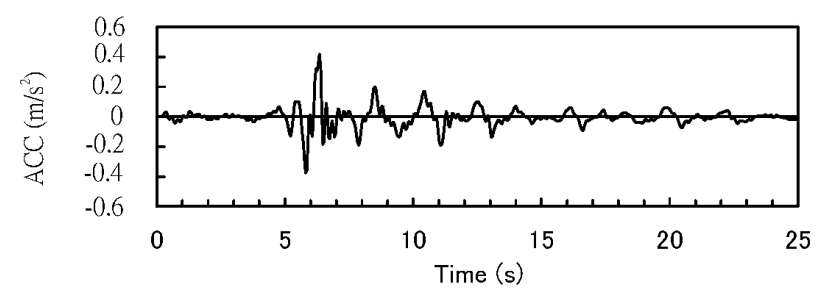

Fig. 4 Seismic wave finite element mesh for Ground I and II and that for Single pile I and II are shown in Figs. 5 and 6, respectively. The size of the ground is $23 \mathrm{~m}$ in length, $11 \mathrm{~m}$ in thickness, and $16.6 \mathrm{~m}$ in height. The number of nodes is 2280, and the number of 8 -node isoparametric elements is 1764 for Ground I and II. Ten more nodes are used for Single pile I and II to represent the superstructure with a nodal mass loaded on top. The 24.9 ton mass is elevated at $5.4 \mathrm{~m}$ above ground surface to represent the position of inertial force in prototype scale test for Single pile I and II, for which the structure is simulated by linear elastic beam bending elements with consistency mass. All of the finite element meshes introduced in here are expressed in a prototype scale.

The boundary conditions are as follows: (a) the bottom of the ground is fixed; (b) the vertical boundaries parallel to the XOZ plane are fixed in the y-direction and free in the $\mathrm{x}$ and $\mathrm{z}$-directions; and (c) an equaldisplacement boundary condition is used between the two-side boundaries, whose normal direction is parallel to the $x$-direction to simulate the infinite boundary in real situation (Kimura and Zhang [10] proved the effectiveness of this method by using a numerical tool). The boundary condition of toe of the pile in the numerical model is fixed in the x-direction as in the physical model tests. No interface elements are employed among any structure and soils in this research. The initial stress condition of the ground is due to the gravity.

In calculating the viscous matrix, an eigenvalue analysis for the full system is conducted to evaluate the first two eigenvalues. The eigenvalue analysis conducted with a hybrid of Jacobian and subspace methods shows that the first two eigen-periods are $0.803 \mathrm{sec}$ and $0.448 \mathrm{sec}$ for the ground with unit weight of $14.3 \mathrm{kN} / \mathrm{m}^{3}$ and $0.766 \mathrm{sec}$ and $0.431 \mathrm{sec}$ for the ground with unit weight of $15.8 \mathrm{kN} / \mathrm{m}^{3}$. However, it should be pointed out that in the case of nonlinear analysis, eigenvalue analysis is only related to the initial stiffness. A Rayleigh-type damping is adopted, and the damping coefficients of the structures and the ground are assumed to be $2 \%$ and $10 \%$, respectively, in the dynamic analysis of the full system. Although the stiffness of the ground, the piles, and the upper structure may change because of the nonlinearity of these materials, the viscous matrix calculated from the Rayleigh-type damping is assumed to be constant irrespective of the changes in the stiffness matrix in this study.

The tij model parameters for Toyoura standard sand are well defined in many related studies published in Japan, and they are, except for the stress-dilatancy parameter $\alpha$, obtained in static loading tests. However, they should be reviewed closely in a dynamic analysis. Therefore, the soil parameters of Ground I and II are first calibrated by centrifugal vibration tests, and then the physical model tests of Single pile I and II are simulated using the same calibrated parameters. Accuracy and consistency of the applied numerical tool can thus be proven after such process. 


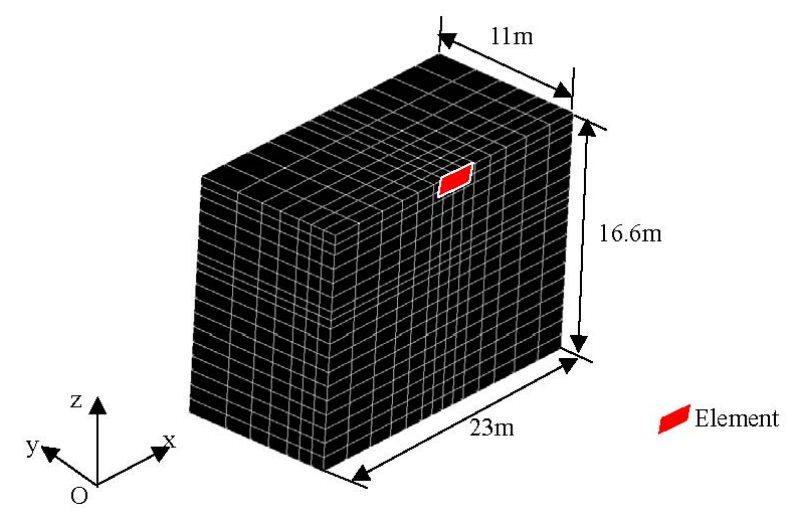

Fig. 5 Finite element mesh for Ground I and II

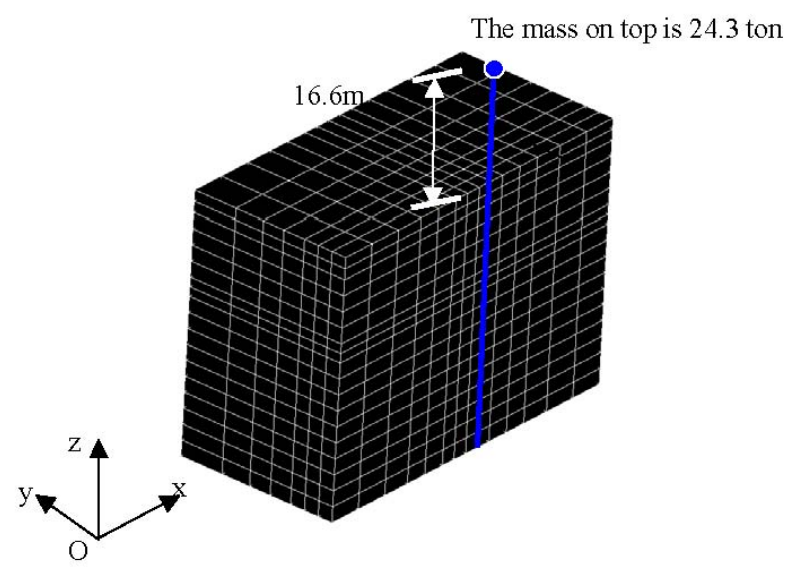

Fig. 6 Finite element mesh for Single pile I and II

\section{RESULTS AND DISCUSSIONS}

\subsection{Ground I and II Cases}

The response acceleration time histories for Ground I and II are plotted in Figs. 7 and 8, respectively. The numerical results agree well with the physical model test measurements obtained at ACC4, ACC3, and ACC2 located at $3.51 \mathrm{~m}, 5.76 \mathrm{~m}$ and $8.46 \mathrm{~m}$ beneath the ground surface in prototype scale. A reasonable agreement between the computed and the observed results in those figures shows that the equal-displacement boundary condition used between the two-side boundaries is able to keep the marked points away from the disturbances induced by the two-side boundaries in the numerical simulation. This type of numerical boundary condition is a more efficient but simple way to take into account the boundary effect in coding a dynamic FE analysis than absorbing boundary condition that may be more complex. However, the reason causing the obvious discrepancy of magnitudes and time of peak accelerations could be the soil model over estimated the stiffness of the grounds during phase transformation.

Stress-strain relationship of the soil elements which is $5 \mathrm{~m}$ beneath ground surface in Ground I and II are reproduced in Fig. 9 using the numerical tool. It illustrates that the deformation of the softer ground is larger
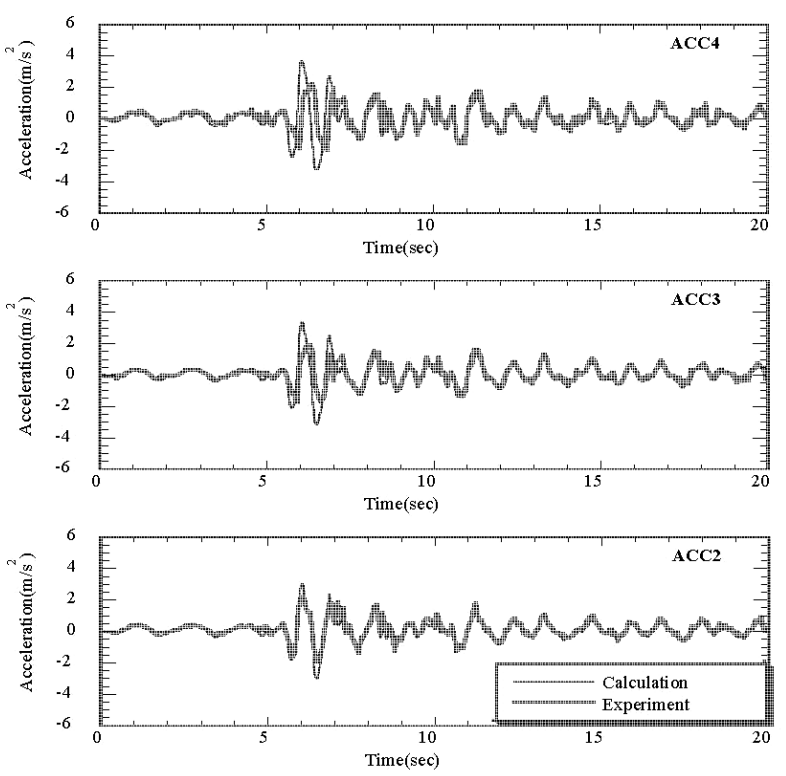

Fig. 7 Time history of acceleration of Ground I
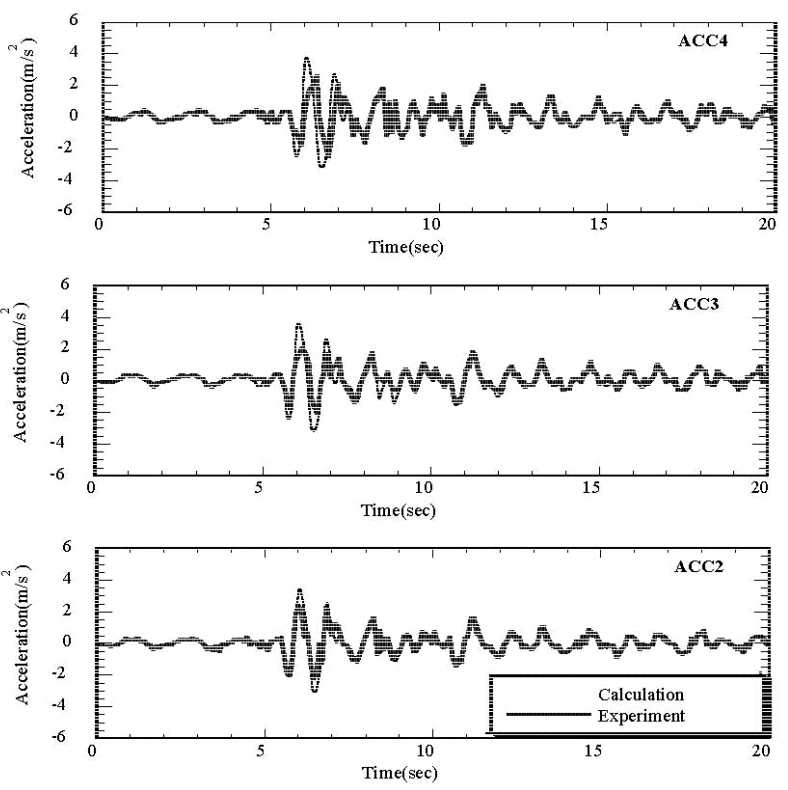

Fig. 8 Time history of acceleration of Ground II

than that of the stiffer one. Also, the two hysteretic loops show that the dynamic response of the soils has reached plastic state in this seismic event and that the attention must be paid to the need for an appropriate elasto-plastic model in order to take into account soil behaviors in large vibration than just a simple model. Although the numerical simulation for ground behavior under a major earthquake should consider the nonlinearity due to changing stiffness of ground, a constant viscous matrix is proven to be effective.

\subsection{Single Pile I and II Cases}

The response acceleration time histories for Single pile I and II are plotted in Figs. 10 and 11, respectively. The physical model test measurements were obtained at 

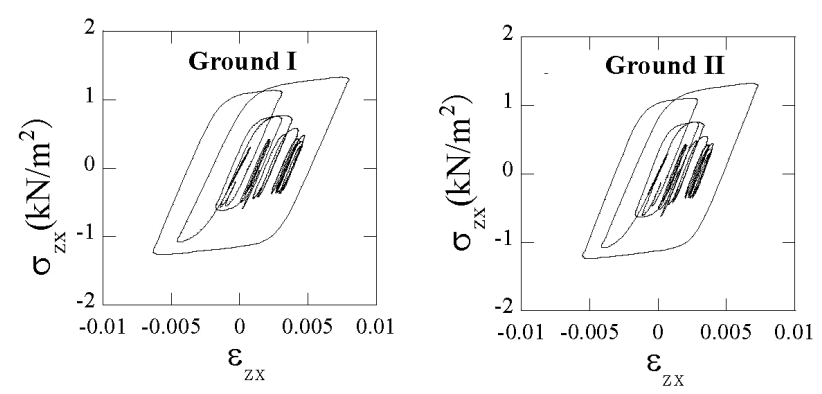

Fig. 9 Computed stress-strain relationship of Ground I and Ground II
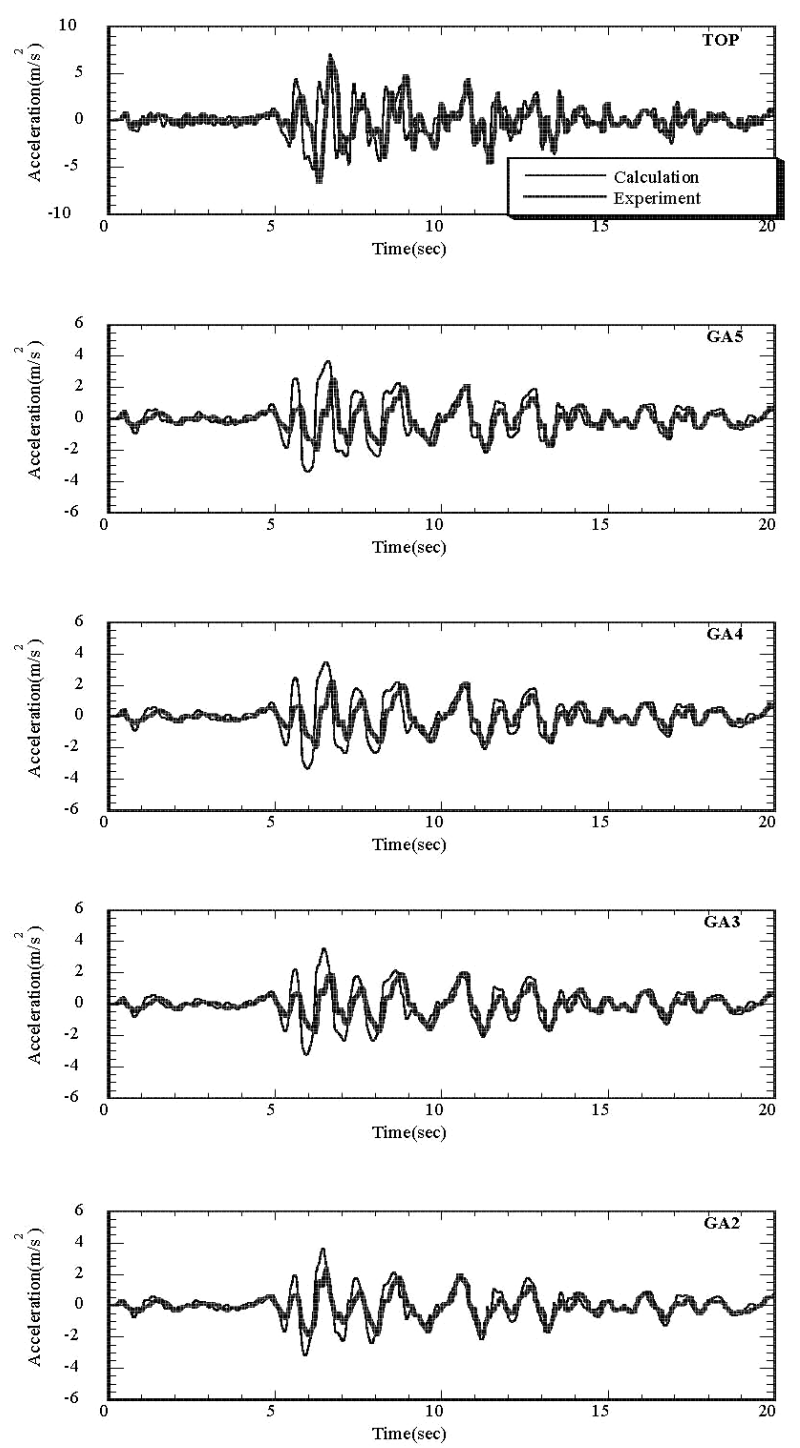

Fig. 10 Time history of acceleration of Single pile I

GA5, GA4, GA3 and GA2 located at $1.26 \mathrm{~m}, 3.51 \mathrm{~m}$, $5.76 \mathrm{~m}$, and $8.46 \mathrm{~m}$ beneath the ground surface in prototype scale. It is evident that except from $5 \mathrm{sec}$ to $10 \mathrm{sec}$, the numerical prediction of the accelerations for Single pile I is relatively accurate at most of the time when compared to the physical test results. The discrepancy may be due to the pile volume effect in the physical
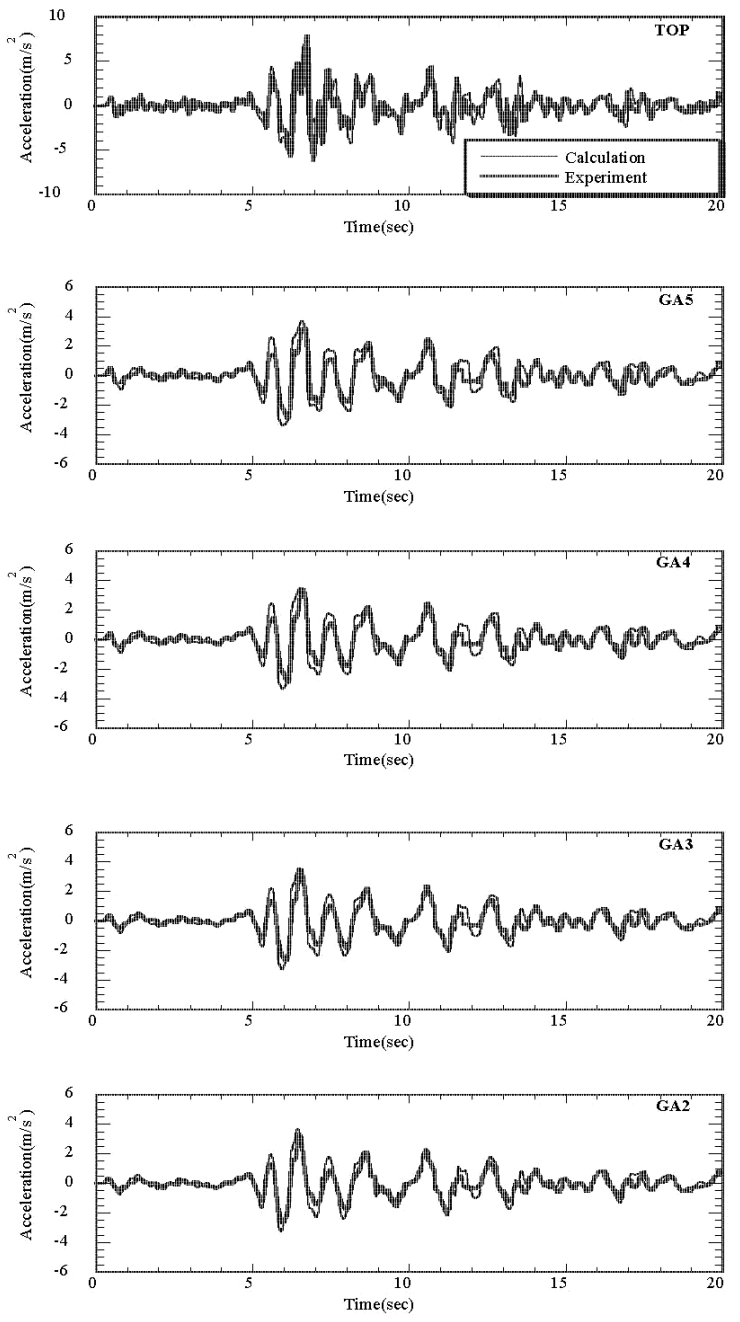

Fig. 11 Time history of acceleration of Single pile II

model test that is not considered in the numerical model, and it shows that the volume of pile has more significant effect in the softer ground and during the period of large vibration. On the other hand, the pile volume may not affect as much in the case of Single pile II in which the ground is consisted of denser sandy soils. However, it is necessary to point out that the computed and the observed response accelerations agree well with each other on the top of Single pile I and II regardless of the pile volume effect.

The bending moment time histories for Single pile I and II are plotted in Figs. 12 and 13, respectively. The physical model test results were obtained from BM4, $\mathrm{BM} 3, \mathrm{BM} 2$, and BM1 located at $1.26 \mathrm{~m}, 3.51 \mathrm{~m}, 5.76 \mathrm{~m}$, and $8.46 \mathrm{~m}$ beneath the ground surface in prototype scale. It can be seen from the figures that the calculated bending moments agree relatively well with the observed ones mostly during the time period of less than $7 \mathrm{sec}$. It may be the reason that the damping of the prototype structure was different from that used in the analysis.

\subsection{Further Analysis of the Results}

Comparing the bending moment time histories for Single pile I and II, the amplitude of the computed and 

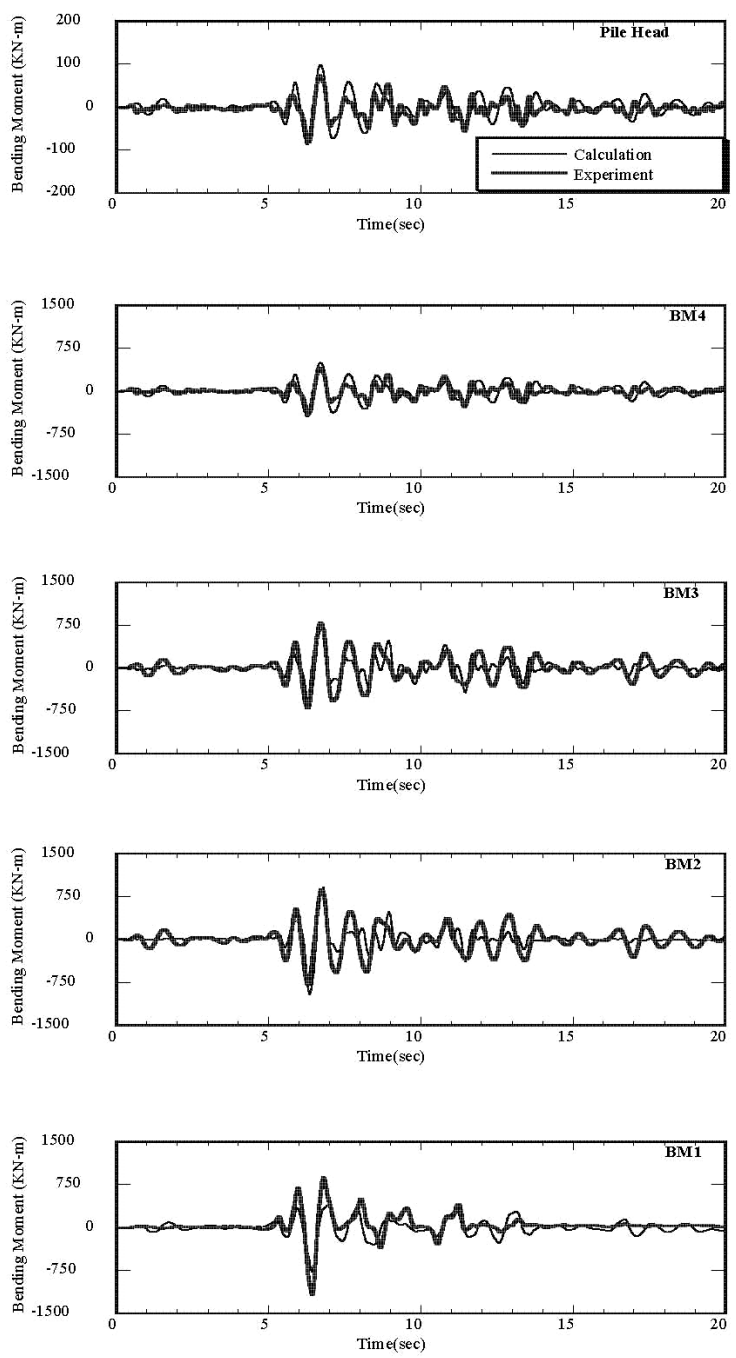

Fig. 12 Time history of the bending moment of pile in the case of Single pile I

the measured bending moments on pile head in Single pile II is larger than that in Single pile I due to the denser ground. The results also provide some level of confidence for the parameters employed and the numerical techniques applied in the analysis, and it indicates indirect validity for the experimental and the measurement techniques.

It needs to be pointed out again that the pile in the physical models is represented numerically by beam element that is not able to take into account either the actual shape or the volume of the pile in a numerical model. The comparisons between the computed and the observed responses of both bending moment and acceleration of the pile demonstrate the validity of employing the method with some level of confidence. However, the volume and shape of pile must be considered if the attention in future study is paid on the soils closer to the pile foundation or/and in a relative soft ground, and the elements such as plate element, shell element, or solid element could be used to represent pile volume if more accurate bending moment responses of the pile are to be obtained.
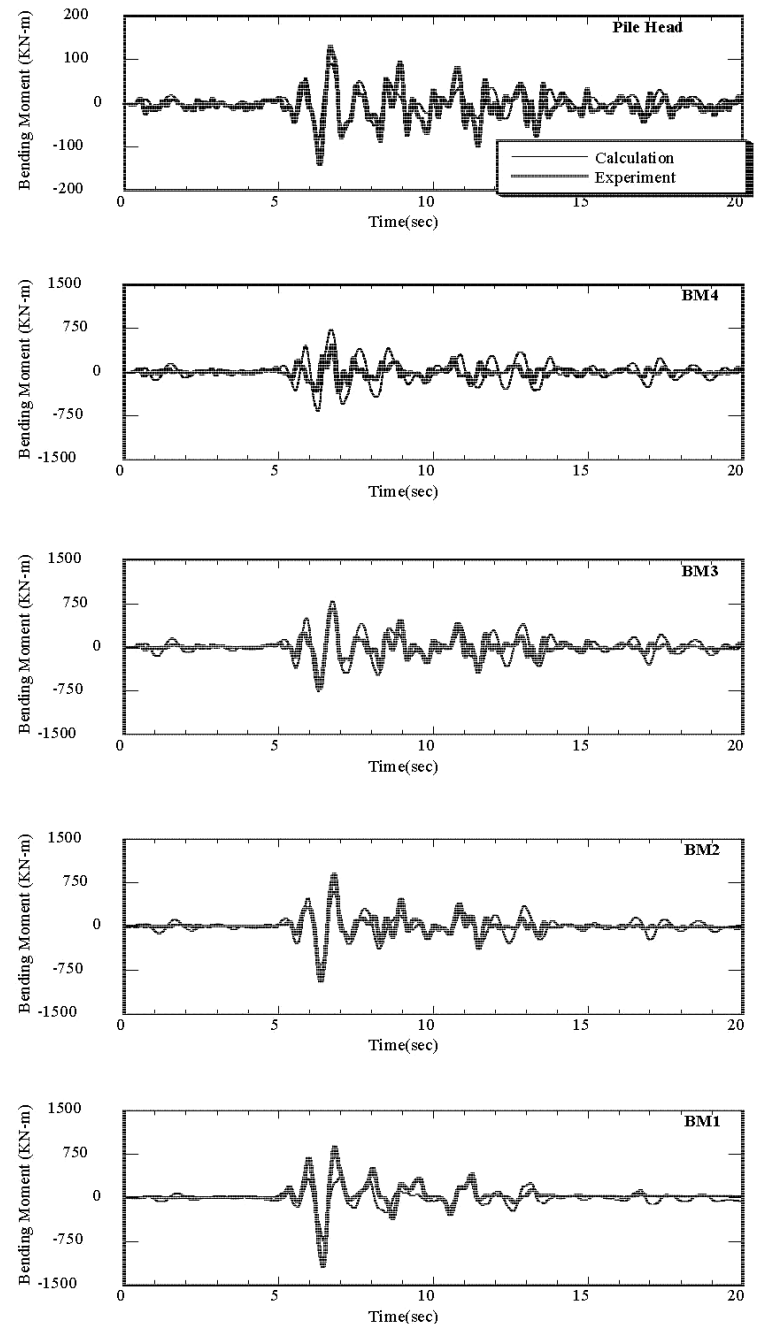

Fig. 13 Time history of the bending moment of pile in the case of Single pile II

Also, soil density is modeled as the control factor in this paper, and it was observed that simulations for Ground II and Single pile II that are installed in denser sandy ground exhibit a better agreement than that of Ground I and Single pile I with looser sandy ground. The reasons for such results could be (1) the limitations of the soil model for prediction of behavior of loose sandy soils or (2) the density control effect for loose sandy ground in the physical model tests.

\section{CONCLUSIONS}

By comparing the results of a numerical threedimensional analysis to that of centrifuge tests, the following conclusions are obtained.

1. By examining the proposed numerical tool using a series of physical tests, the numerical code is proven to be consistent, and the soil model parameters are confirmed in a rigorous way.

2. The numerical predictions of Ground I and Single 
pile I, of which the ground consists of softer soil, and Ground II and Single pile II, of which the ground consists of harder soil, exhibit a reasonable agreement with the experimental work. The results show that the employed soil constitutive model, tij sand model, is able to properly reproduce seismic response of different grounds.

3. In this study, methodologies that employ constant viscous matrix and equal-displacement boundary condition in dealing with a complicated dynamic analysis for soil-pile-superstructure system are explained, and their effectiveness is demonstrated by comparing with the physical model tests.

4. It is possible to use a beam element for pile representation without considering its shape and volume in a numerical simulation to measure the response acceleration when attention is paid on the pile head and on the soil away from the pile foundation. However, when attention is paid on the soil close to the pile, which is thought to be strongly influenced by the pile foundation especially when the grounds are soft, the shape and volume of pile should be considered with a good consideration in future study.

5. With an assumption that the pile and the surrounding soil bond perfectly with each other during shaking, no interface element was employed in the study. At the level of shaking employed in this study, such assumption may be acceptable.

6. The numerical tool is better at simulating the models with denser sandy soils than those with soft ones possibly due to the limitations of the soil model and/or the density control effect on loose sandy soils.

\section{ACKNOWLEDGEMENTS}

This author would like to express his sincere thanks to Prof. Makoto Kimura in Kyoto University, Japan and Prof. Feng Zhang in Nagoya Institute of Technology, Japan for their valuable support on obtaining the experiment data and suggestions. The author also would like to thank three anonymous referees for providing constructive and inspiring review.

\section{REFERENCES}

1. EI-Masrafawi, H., Kaynia, A. M. and Novak. M., "The Superposition Approach to Pile Group Dynamics," Geot. Special Publication, ASCE, 34, pp. 114-135 (1992).

2. Anandarajah, A., Rashidi, H. and Arulanandan, K., "Elasto-Plastic Finite Element Analysis of a SoilStructure System Under Earthquake Excitation," Computers and Geotechnics, 17, pp. 301-325 (1995).

3. Fukutake, K., Ohtsuki, A. and Yoshimi, Y., "A New Soil Cement Block System for Protecting
Piled in Liquefiable Ground," Proc. of 1st Int. Conf. on Earthquake Geotechnical Engineering, Tokyo, Japan, pp. 605-610 (1995).

4. Finn, W. D. L, Thavaraj, T. and Wu, G., "Nonlinear Seismic Response of Pile Foundations during Strong Earthquake Shaking," Proc. of Int. Symp. Seismic Behavior of Ground and Geotechnical Structure, Rotterdam, The Netherlands, pp. 195-204 (1997).

5. Wakai, A., Ugai, K. and Matsuda, T., "Seismic Analysis of a Bridge-Ground System Excited in the Axial Direction of the Bridge," Proc. of $6^{\text {th }}$ Int. Symp. Numerical Models in Geomechanics, Montreal, Canada, pp. 709-712 (1997).

6. Imamura, S., Hagiwara, T., Tsukamoto, Y. and Ishihara, K., "Response of Pile Groups Against Seismically Induced Lateral Flow in Centrifuge Model Tests," Soils and Foundations, Japanese Society of Soil Mechanics and Foundation Engineering, 44(3), pp. 39-55 (2004).

7. Lin, S. S., Liao, J. C., Chen, J. T. and Chen, L., "Lateral Performance of Piles Evaluated via Inclinometer Data," Computer and Geotechnics, 32, pp. 411-421 (2005).

8. Tokimatsu, K., Suzuki, H. and Sato, M., "Effects of Inertial and Kinematic Interaction on Seismic Behavior of Pile with Embedded Foundation," Soil Dynamics and Earthquake Engineering, 25, pp. 753-762 (2005).

9. Finn, W. D. L., Thavaraj, T., Wilson, D. W., Boulanger, R. W. and Kutter, B. L., "Seismic Response Analysis of Pile Foundations at Liquefiable Sites," Proc. $7^{\text {th }}$ US-Japan Workshop on Earthquake Resistant Design of Lifeline Facilities and Countermeasures Against Liquefaction, Technical Report, Seattle, USA, pp. 579-586 (1999).

10. Kimura, M. and Zhang, F., "Seismic Evaluations of Pile-Foundations with Three Different Methods Based on Three-Dimensional Elasto-Plastic Finite Element Analysis," Soils and Foundations, Japanese Society of Soil Mechanics and Foundation Engineering, 40, pp. 113-132 (2000).

11. Zhang, F., Kimura, M., Nakai, T. and Hoshikawa, T., "Mechanical Behavior of Pile Foundations Subjected to Cyclic Lateral Loading Up to the Ultimate State," Soils and Foundations, Japanese Society of Soil Mechanics and Foundation Engineering, 40, pp. 1-17 (2000).

12. Ye, G. L., Miyaguchi, H., Huang, Yu, Sawada, H., Zhang, F. and Yashima, A., "Dynamic behavior of group-pile foundation evaluated by simplified model and sophisticated model," Proc. 13th WCEE, Vancouver, Canada, Paper 28, CD-ROM, (2004).

13. Nakai, T., "An Isotropic Hardening Elastoplastic Model for Sand Considering the Stress Path Dependency in Three-Dimensional Stresses," Soils and Foundations, Japanese Society of Soil Me- 
chanics and Foundation Engineering, 29, pp. 119-137 (1989).

14. Chowdhury E Q., "Elastoplastic Models for Clays Under Monotonic and Cyclic Loading Using Conventional and Modified Stresses," Doctoral Dissertation, Nagoya Institute of Technology, Japan (1998).

15. Nakai, T. and Hinokio, M., "A Simple Elastoplasic Model for Geomaterials Based on TIJ-Concept and Its Calibration by Various Test Data," Proc. of Sessions of the Geo-Frontiers 2005 Congress, Texas, USA, pp. 455-465 (2005)

16. Farias, M. M., Nakai, T., Shahin, H. M., Pedroso, D. H., Passos, P. G. O. and Hinokio, M., "Ground Densification Due to Sand Compaction Piles," Soils and Foundations, Japanese Society of Soil Mechanics and Foundation Engineering, 45, pp. 214230 (2005)

17. Schofield, A. N., "Centrifuge Test," Soil Dynamics and Earthquake Engineering, 2, pp. 183-187 (1983).
18. Scott, R. F., "Centrifuge Model Testing at Caltech," Soil Dynamics and Earthquake Engineering, 2, pp. 185-198 (1983).

19. Prevost, J. H. and Scanlan, R. H., "Dynamic SoilStructure Interaction: Centrifuge Modeling," Soil Dynamics and Earthquake Engineering, 2, pp. 212-221 (1983).

20. Oogawa, S., Kameii, H., Zhang, F. and Kimura, M., "A Study on Seismic behavior of Jacket-type Foundation Using Centrifuge Test (I)," Proc. of 26th Earthquake Engineering Symposium, Japanese Society of Soil Mechanics and Foundation Engineering, pp. 56-61 (2001) (in Japanese).

(Manuscript received April 25, 2006, accepted for publication July 31, 2007.) 
